\title{
Graphical description of Pauli measurements on stabilizer states
}

\author{
Matthew B. Elliott* \\ Department of Physics and Astronomy, MSC07-4220, \\ University of New Mexico, Albuquerque, NM 87131-0001 \\ Bryan Eastin \\ National Institute of Standards and Technology, Boulder, CO 80305 \\ Carlton M. Caves \\ Department of Physics and Astronomy, MSC07-4220, \\ University of New Mexico, Albuquerque, NM 87131-0001 and \\ Department of Physics, University of Queensland, Brisbane, QLD 4066, Australia
}

\begin{abstract}
We use a graphical representation of stabilizer states to describe, simply and efficiently, the effect of measurements of Pauli products on stabilizer states. This work complements our earlier work [Phys. Rev. A 77, 042307 (2008)], which described in graphical terms the action of Clifford operations on stabilizer states.

PACS numbers: 03.67.-a
\end{abstract}

\section{INTRODUCTION}

Recently we introduced a graphical representation of stabilizer states and translated the action of Clifford operations on stabilizer states into graph operations on stabilizer-state graphs [1]. The purpose of that paper was, in part, to augment the stabilizer formalism by providing techniques for understanding and manipulating this important class of states. The purpose of this paper is to extend our previous results by describing graphically the effect of measurements of Pauli products on stabilizer states [2]. ${ }^{1}$

Pauli measurements are the natural set of measurements to consider in the context of stabilizer states, because the post-measurement state, as an eigenstate of the measured Pauli product, is also a stabilizer state. Since we can represent both the pre- and post-measurement states by graphs, the effect of the measurement can be represented by a graph transformation.

Section II reviews the concept of stabilizer-state graphs and lists some relevant results from Ref. [1]. This is not meant to be a complete introduction to stabilizerstate graphs and is surely insufficient background to enable their comfortable manipulation. Nonetheless, anyone comfortable with stabilizer states and quantum circuits should find the review here sufficient for the needs of this paper and can consult Ref. [1] and references therein for further details. Section III lays out our graphical results for Pauli measurements on stabilizer states and illustrates the results with example measurements. A detailed proof of the measurement transformation is given in the Appendix.

\footnotetext{
*Electronic address: mabellio@unm.edu

${ }^{1}$ See also Ref. [3] for a single-qubit measurement rule applied to a very different graphical representation of stabilizer states.
}

\section{BACKGROUND}

\section{A. Stabilizer-state graphs}

We now review the stabilizer-graph formalism presented in Ref. [1]. Of central importance to the graphical representation of stabilizer states is the fact that all stabilizer states are equivalent under local Clifford operations to some graph state [4, 5]. Furthermore, the conversion of a graph state to any equivalent stabilizer state can be achieved by applying to each qubit a single operation from the following set: $I, Z, H, S, H Z$, and $S Z$ where the gates $I, Z, H, S$ are the identity, sign-flip, Hadamard, and phase gates, respectively.

As a consequence of these facts, one can draw a graph to represent any stabilizer state by first drawing the graph corresponding to a local-Clifford-equivalent graph state and then adding features indicating which local gates must be applied to each qubit of the graph state to transform it into the desired stabilizer state.

Simple graphs, those consisting of solid nodes connected by edges, are used to represent graph states in the standard way $[2,5]$. In terms of a preparation circuit, this amounts to associating with each node a qubit initially prepared in the state $H|0\rangle$ and associating with each edge a subsequent controlled-sign gate, ${ }^{C} Z$, between the qubits corresponding to the connected nodes. ${ }^{2}$ Stabilizer-graph notation augments this description by representing additional, terminal gates as follows: the application of a $Z$ gate is represented by a node with a negative sign, the application of an $S$ gate by a self loop, and the application an $H$ gate by a hollow node. If present, $H$ gates are assumed to act last; a hollow node with a negative

\footnotetext{
${ }^{2}{ }^{C} Z$ is often called the controlled-phase or controlled- $Z$ gate.
} 
sign thus indicates the application of a $Z$ gate followed by an $H$ gate. In order to associate a stabilizer state with any arrangement of solid and hollow nodes with arbitrary edges and with or without self loops and signs, we choose to interpret a hollow node with a loop as representing the application of an $S$ gate followed by an $H$ gate. Although hollow nodes with loops are not necessary to represent all stabilizer states, they are a beneficial addition when considering the action of local Clifford gates. ${ }^{3}$

Given a stabilizer-state graph on $n$ nodes, we can easily write down the associated $n$-qubit stabilizer state as follows. Let $\mathcal{H}$ denote the set of hollow nodes, $\mathcal{S}$ the set of nodes with loops, and $\mathcal{Z}$ the set of nodes with negative signs. We label by $\Gamma$ the adjacency matrix of the underlying graph, by which we mean the $n \times n$ square matrix whose entries are determined from the graph by

$$
\Gamma_{j k}= \begin{cases}0, & \text { if } j=k \text { or if } j \neq k \text { are not connected } \\ 1, & \text { if } j \neq k \text { are connected }\end{cases}
$$

The associated stabilizer state $|\psi\rangle$ is given by the formula

$$
|\psi\rangle=\prod_{m \in \mathcal{H}} H_{m} \prod_{l \in \mathcal{S}} S_{l} \prod_{k \in \mathcal{Z}} Z_{k} \prod_{i, j}\left({ }^{C} Z_{i j}\right)^{\Gamma_{i j}} H^{\otimes n}|0\rangle^{\otimes n}
$$

where each gate acts upon the qubit(s) identified by its subscript(s).

As a final note, since qubits of a stabilizer state correspond to nodes of a graph, we use the terms "qubit" and "node" interchangeably. Thus we speak of qubits in a graph and of applying Clifford operations to nodes.

\section{B. Graph terminology}

Much of what follows concerns the manipulation of stabilizer-state graphs. In preparation, this subsection introduces a variety of terms describing graph transformations. Some of these terms were adopted from graph theory, while others have been invented for the task at hand.

Among those terms common to graph theory are neighbors, complement, and local complement. The neighbors of a node $j$, which make a set denoted by $\mathcal{N}(j)$, are those nodes connected to $j$ by edges. In the results that follow, a loop does not count as an edge, so a node is never its own neighbor. Complementing the edge between two nodes removes the edge if one is present and adds one otherwise. A local complement is performed by complementing a selection of edges, with the pattern of edges

\footnotetext{
3 To represent all stabilizer states, it is also unnecessary to include graphs in which any hollow nodes are connected by edges (for discussion, see Ref. [1]), but we considered such graphs in Ref. [1] and allow them in our discussion in this paper.
}

depending on whether local complementation is applied to a node or along an edge.

Local complementation on a node complements the edges between all of the node's neighbors. Local complementation along an edge is equivalent to a sequence of local complementations on the nodes defining the edge. This sequence is as follows: first perform local complementation on one of the nodes, then local complement on the other node, and finally local complement on the first node again. Local complementation along an edge is symmetric in the two nodes defining the edge, so it does not matter at which node local complementation is first performed.

To these terms we add flip and advance. Flip is used to describe the simple reversal of some binary property, such as the sign of a node or its fill, i.e., whether the node is solid or hollow. Advance refers specifically to an action on loops; advancing generates a loop on nodes where there was not previously one, and it removes the loop and flips the sign on nodes where there was a loop. Its action mirrors the application of the phase gate, since $S^{2}=Z$.

\section{Graphical description of Clifford operations}

We make use of the following transformation rules [1], which constitute a graphical description of the action of $H, S$, and $Z$ gates on stabilizer states.

T1. Applying $H$ to a node flips its fill.

T2. Applying $S$ to a solid node advances its loop.

T3. Applying $S$ to a hollow node without a loop performs local complementation on the node and advances the loops of its neighbors.

If the node has a negative sign, flip the signs of its neighbors as well.

T4. Applying $S$ to a hollow node with a loop flips its fill, removes its loop, performs local complementation on it, and advances the loops of its neighbors.

If the node does not have a negative sign, flip the signs of its neighbors as well.

T5. Applying $Z$ to a solid node flips its sign.

T6. Applying $Z$ to a hollow node flips the signs of all of its neighbors. If the node has a loop, its own sign is flipped as well.

\section{Equivalent graphs}

Two graphs that look different can represent the same stabilizer state. Because of this fact, we make use of the following equivalence rules. 
E1. Flip the fill of a node with a loop. Perform local complementation on the node, and advance the loops of its neighbors.

Flip the node's sign, and if the node now has a negative sign, flip the signs of its neighbors as well.

E2. Flip the fills of two connected nodes without loops, and local complement along the edge between them.

Flip the signs of nodes connected to both of the two original nodes. If either of the two original nodes has a negative sign, flip it and the signs of its current neighbors.

Applying these equivalence rules to a stabilizer-state graph results in a (generally different) graph that represents the same stabilizer state. In fact, successive application of these rules generates all graphs corresponding to a given state.

\section{GRAPHICAL DESCRIPTION OF PAULI MEASUREMENTS}

We now turn to our graphical formulation of Pauli measurements on stabilizer states.

Let $M$ be an $n$-fold tensor product of the identity, $I$, and the Pauli matrices, $X, Y$, and $Z$, that is,

$$
M=\bigotimes_{j=1}^{n} M_{j},
$$

where $M_{j}=I, X, Y$, or $Z$. If $M_{j} \neq I$, we call node $j$ a measured node; otherwise, if $M_{j}=I$, we say the node is not measured.

Given such a measurement operator, $M$, our task is twofold: first, to find the probability that a measurement of $M$ on a quantum system in the stabilizer state $|\psi\rangle$ gives an outcome $(-1)^{a}$, and, second, to determine the post-measurement quantum state of the system, i.e., a post-measurement stabilizer-state graph. This section describes a general graphical rule, applicable to the graph that represents the stabilizer state, which accomplishes these tasks.

\section{A. Simplifying the measurement}

By means of the graph transformation and equivalence rules reviewed in Sec. II, it is possible to greatly reduce the difficulty of formulating a Pauli-measurement rule. The following three paragraphs describe a sequence of three simplifications that can be made to any measurement, thereby restricting its form to one more amenable to a measurement transformation rule.

The first simplification relies on the fact that a measurement where $M_{j}=C Z C^{\dagger}$ is equivalent to a measurement where $M_{j}=Z$ preceded by application of the local Clifford operation $C_{j}^{\dagger}$ and followed by application of $C_{j}$ to the post-measurement state. If $M_{j}=X$, the local Clifford operation needed is $C=H$; if $M_{j}=Y$, it is $C=S H$. Thus, the first simplification is to transform the original graph, using rules T1-T6, so that on the new graph the measurement becomes a product of $Z \mathrm{~s}$ on the measured nodes. This means that it suffices to determine the effect of $Z$-type measurements, that is, measurements with the property that $M_{j}=I$ or $Z$ for all $j$. The post-measurement state must be transformed by application of the appropriate local unitaries to the measured nodes, i.e., $C_{j}$ to measured node $j$; in terms of graphs, this post-measurement transformation is handled by rules $\mathrm{T} 1-\mathrm{T} 4$.

The second simplification is to reduce the graph, a procedure introduced in Ref. [1]. A reduced graph is one in which hollow nodes are loopless and unconnected to one another. Any stabilizer state can be represented by a reduced graph. Given a stabilizer state represented by a stabilizer graph, one can find an equivalent reduced graph by applying equivalence rules E1 and E2 to the given graph. One applies rule E1 to any hollow node with a loop and uses E2 on any pair of connected hollow nodes without loops. Each application of E1 or E2 makes solid the node(s) it is applied to, without introducing new hollow nodes, so the procedure terminates in a reduced graph. For the purposes of our measurement analysis, we are only required to reduce the measured nodes, not the entire graph, so the procedure terminates in a number of iterations that does not exceed the number of measured nodes. After this second simplification, there are no loops on hollow measured nodes and no edges between hollow measured nodes.

The final simplification is to disconnect hollow measured nodes from unmeasured nodes by using equivalence rules E1 and E2. Suppose that a measured hollow node is connected to an unmeasured node. In the case that the unmeasured node does not have a loop, applying equivalence rule E2 to the pair turns the measured node solid. If the unmeasured node has a loop, an application of E1 to the unmeasured node gives the measured hollow node a loop. Now one can apply E1 to the measured hollow node to turn it solid. One can verify that in both cases, application of the equivalence rules leaves the remaining measured hollow nodes loopless and unconnected to one another. Thus, this last simplification terminates in a number of iterations no greater than the number of measured hollow nodes.

The end product of these simplifications is a $Z$-type Pauli measurement on a graph in which measured hollow nodes are loopless and unconnected to one another and to unmeasured nodes. 


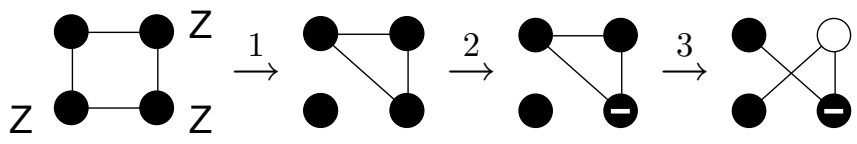

FIG. 1: The steps that perform a Pauli product measurement $M=I \otimes Z \otimes Z \otimes Z$ on a stabilizer-state graph for the fourqubit cluster state. The juxtaposition of Pauli operator and node indicates the presence of that operator in the intended measurement. We label the nodes in the graphs clockwise starting from 1 in the upper left corner. Thus, $\mathcal{M}=\{2,3,4\}$, $\mathcal{M}_{\mathrm{S}}=\mathcal{M}_{\mathrm{SE}}=\{2,3,4\}$, and $\mathcal{M}_{\mathrm{H}}=\varnothing$. Since $\mathcal{M}_{\mathrm{SE}} \neq \varnothing$, the outcome is random; for the sake of illustration, we take it to be +1 . Node 2 is taken to be the chosen node, so the edges between its neighbors, nodes $\{1,3\}$, and the unchosen nodes in $\mathcal{M}_{\mathrm{SE}}$, nodes $\{3,4\}$, are complemented in step 1 . The chosen node does not have a sign, and $a+b$ is even if we assume $\mathrm{a}+1$ measurement outcome, so step 2 has the effect of giving a sign to the node in $\mathcal{M}_{\mathrm{SE}}$ that is also a neighbor of node 2 , meaning node 3 . Step 3 removes all edges involving node 2 and then connects it to nodes 3 and 4 while making node 2 hollow. Finally, step 4 has no effect since the chosen node has no loop. If either node 3 or 4 had been picked as the chosen node, the resulting graph could be transformed into this one by using equivalence rule E2.

\section{B. Graphical description of simplified measurements}

With the preceding simplifications carried out, we can now spell out the graphical description of the measurement. The proof of this description is given in terms of circuit identities in the Appendix.

In addition to the sets $\mathcal{H}, \mathcal{S}$, and $\mathcal{Z}$ introduced in Sec. II A, we require the use of several other sets of nodes in the stabilizer-state graph: $\mathcal{M}=\left\{j \mid M_{j} \neq I\right\}$ is the set of measured nodes, $\mathcal{M}_{\mathrm{S}}=\mathcal{M} \backslash \mathcal{H}$ is the set of measured solid nodes, $\mathcal{M}_{\mathrm{H}}=\mathcal{M} \cap \mathcal{H}$ is the set of measured hollow nodes, and $\mathcal{M}_{\mathrm{SE}}=\left\{j \in \mathcal{M}_{\mathrm{S}}|| \mathcal{M}_{\mathrm{H}} \cap \mathcal{N}(j) \mid=\right.$ $0(\bmod 2)\}$ is the set of measured solid nodes that have an even number of connections to measured hollow nodes. Here $\mathcal{A} \backslash \mathcal{B}$ denotes the set of elements in $\mathcal{A}$ that are not in $\mathcal{B}, \mathcal{A} \cap \mathcal{B}$ denotes the intersection of $\mathcal{A}$ and $\mathcal{B}$, and $|\mathcal{A}|$ denotes the number of elements in $\mathcal{A}$.

When a Pauli measurement is made on a stabilizer state, the outcome is either random, with the two possible outcomes being equiprobable, or certain. Which case applies depends on $\mathcal{M}_{\mathrm{SE}}$. The outcome in the deterministic case is specified by

$$
b=\left|\mathcal{M}_{\mathrm{H}} \cap \mathcal{Z}\right|,
$$

the number of measured hollow nodes with a sign.

The result of a $Z$-type Pauli measurement is as follows.

1. If $\mathcal{M}_{\mathrm{SE}}=\varnothing$, the measurement outcome is $(-1)^{b}$ with certainty, and the state is unchanged by the measurement.

2. If $\mathcal{M}_{\mathrm{SE}} \neq \varnothing$, the measurement outcome, $(-1)^{a}$, is random, and a graph for the post-measurement state can be obtained according to steps 1-4 below.
To find the post-measurement state when $\mathcal{M}_{\mathrm{SE}} \neq \varnothing$, it is necessary first to pick a node, which we call the chosen node, from $\mathcal{M}_{\mathrm{SE}}$. The post-measurement state is then obtained by the following four steps.

1. For each neighbor of the chosen node, complement all of its edges to unchosen nodes in $\mathcal{M}_{\mathrm{SE}}$.

2. If the chosen node has no sign, flip the signs of all its neighbors that are also in $\mathcal{M}_{\mathrm{SE}}$; otherwise, if the chosen node has a sign, remove that sign, and flip the signs of all other nodes in $\mathcal{M}_{\mathrm{SE}}$ that do not neighbor the chosen node. If $a+b$ is odd, flip the signs of the chosen node and all its neighbors.

3. Remove all edges involving the chosen node, and then connect the chosen node to all the other nodes in $\mathcal{M}_{\mathrm{SE}}$. Make the chosen node hollow.

4. If the chosen node has a loop, remove that loop, perform local complementation on the chosen node, advance the loops of its neighbors, and if $a+b$ is odd, flip the signs of the unchosen nodes in $\mathcal{M}_{\mathrm{SE}}$.

These steps constitute a complete graphical description for the effect of the measurement $M$ on the state. Notice that, in step 1, an edge between two nodes that are in $\mathcal{M}_{\mathrm{SE}}$ and are initially neighbors of the chosen node gets complemented twice, so it remains unchanged. Figure 1 illustrates the use of these rules for the case of a threequbit measurement on a four-qubit cluster state.

In the case that $\mathcal{M}_{\mathrm{SE}}$ has a single element, it becomes the chosen node, and the steps in the measurement transformation rule simplify to the following.

$1^{\prime}$. Do nothing. There are no unchosen nodes in $\mathcal{M}_{\mathrm{SE}}$.

$2^{\prime}$. If the chosen node has a sign, remove that sign. If $a+b$ is odd, flip the signs of the chosen node and all of its neighbors.

$3^{\prime}$. Disconnect the chosen node from the graph, and make it hollow.

$4^{\prime}$. If the chosen node has a loop, remove that loop.

These four steps can be summarized as follows: Remove all loops and signs from the chosen node. Flip the signs of the chosen node and its neighbors if $a+b$ is odd. Then disconnect the chosen node from the graph, and make it hollow.

\section{Single-qubit measurements}

Single-qubit measurements are a straightforward but important special case [6], as illustrated, for example, by the use of such measurements in measurement-based quantum computation [7]. In this section, we specialize the measurement transformation rule of the previous subsection to Pauli measurements on a single measured 
(a)

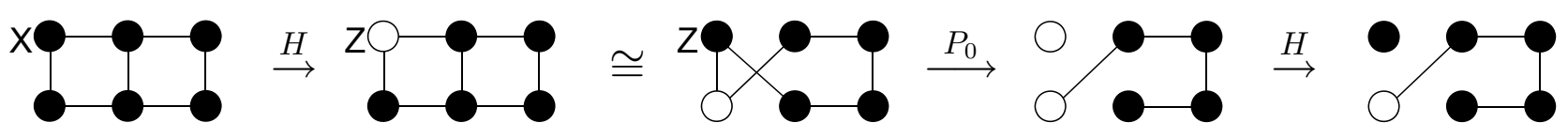

(b)

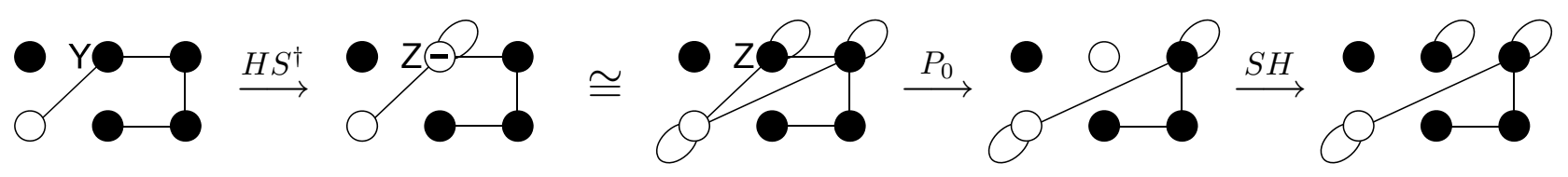

(c)

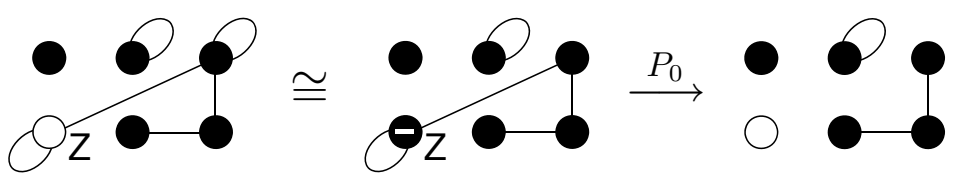

FIG. 2: Examples of the graph manipulations associated with successive single-qubit measurements of (a) $X$, (b) $Y$, and (c) $Z$ on a $2 \times 3$ cluster state. The juxtaposition of Pauli operator and node is used to indicate the intended measurement of that operator on the node. State transformations are indicated by arrows labeled by the transformation being applied to the measured qubit. All $Z$ measurements are assumed to yield outcome +1 , thereby applying the projector $P_{0}$ to the measured qubit. Let the nodes be labeled clockwise starting from 1 in the upper left corner. The measurement of $X_{1}$ in (a) is accomplished by transforming both state and measurement by $H_{1}$, applying equivalence rule E2 to nodes 1 and 6 , applying the measurement transformation to node 1 , and, finally, applying $H_{1}$ to the resultant state. Similarly, the measurement of $Y_{2}$ in (b) is accomplished by transforming both state and measurement by $H_{2} S_{2}^{\dagger}$, applying equivalence rule E1 to node 2 , applying the measurement transformation to node 2, and applying $S_{2} H_{2}$ to the resultant state. The measurement of $Z_{6}$ in (c) requires only the application of equivalence rule E1 to node 6 , followed by application of the measurement transformation to node 6 . In each case the equivalence rule is necessary to fill the node of interest so that our measurement rule for $Z$ can be applied. In parts (a) and (b) it is first necessary to transform the measurement to a $Z$ measurement. By transforming the state as well, we leave the measurement outcome invariant and ensure that the inverse transformation applied after the $Z$ measurement yields the appropriate state. (This caption is a tribute to the late John A. Wheeler and his devotion to writing long, self-explanatory captions.)

node, thereby producing a very simple graphical description of such single-qubit measurements.

In the context of single-qubit measurements, the simplification procedure in Sec. III A ensures that $\mathcal{M}$ consists either of a solid node or of a loopless hollow node that is disconnected from the rest of the graph. In the latter case, the measured qubit is in the state $|0\rangle$ if it has no sign or $|1\rangle$ if it does; the outcome is certain $\left(\mathcal{M}_{\mathrm{SE}}=\varnothing\right)$ and equal to the sign of the hollow node. In the former case, $\mathcal{M}_{\mathrm{SE}}$ consists of the measured solid node, $b=0$, and the outcome $(-1)^{a}$ is random. In this case, steps $1-4$ of Sec. III B, which generate a post-measurement graph, reduce to steps $1^{\prime}-4^{\prime}$ with $b=0$. Thus, the four steps now reduce to the following: Remove all loops and signs from the measured node. Flip the signs of the measured node and its neighbors if the outcome is -1 ( $a$ is odd). Then disconnect the chosen node from the graph, and make it hollow.

We illustrate the transformations associated with single-qubit measurements in Fig. 2. To highlight the relevance of our results to measurement-based quantum computation, successive $X, Y$, and $Z$ measurements on a $2 \times 3$ cluster state are considered. We purposefully follow an inefficient measurement order for pedagogical reasons. In the examples, each measurement outcome is random, and, for purposes of constructing a post-measurement graph, we assume the outcome of each measurement is +1 .

\section{CONCLUSION}

In this paper we have presented a graphical rule for transforming stabilizer states under the measurement of products of Pauli operators both in the general case and in the special case of single-qubit measurements. Together with the transformation rules for Clifford gates given in Ref. [1], the transformation rules for Pauli measurements allow any operation taking one stabilizer state to another to be represented pictorially. Thus, the present paper completes a novel graphical representation of these ubiquitous states.

\section{Acknowledgments}

Thanks to Adam Meier, Manny Knill, and David Smith for their careful readings of this document. The work presented here was supported in part by National Science Foundation Grant No. PHY-0653596. Contributions by NIST, an agency of the US government, are not subject to copyright laws. 
(a)

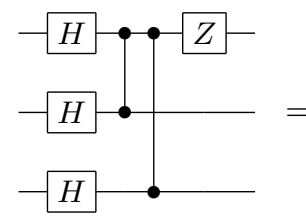

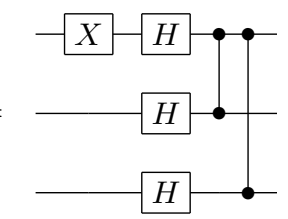

(b)
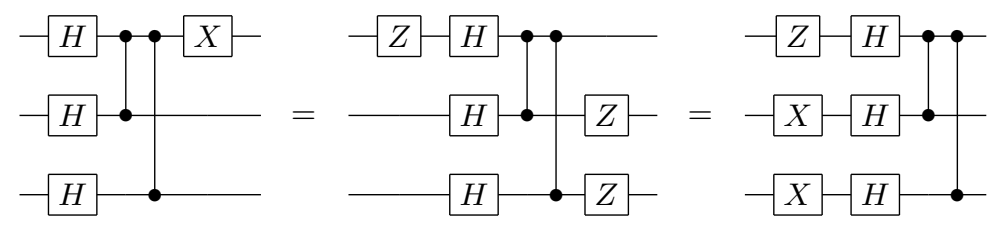

(c)
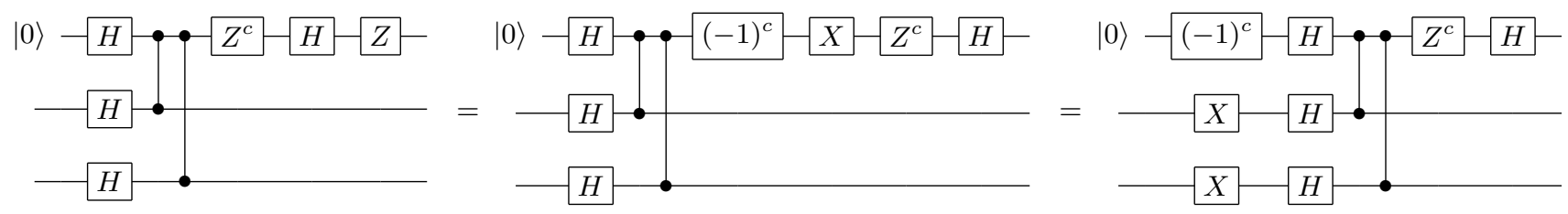

FIG. 3: Circuit identities used to determine the effect of a $Z$ operator on a stabilizer state. Identity (a) follows from the fact that $Z$ and controlled-sign gates commute and from the identity $H Z H=X$. A similar identity holds if the node has a loop or a sign since $Z$ commutes with itself and with $S$. The first equality in identity (b) is easily verified in the standard basis, and the second equality is an application of (a). Identity (c) follows from the equalities shown; the second equality uses the identity in (b).

\section{APPENDIX: PROOF OF THE GENERAL CASE}

In this Appendix we derive the measurement rule given in Sec. III B for transforming a stabilizer graph under a measurement $M$, where $M$ is any tensor product of $I$ and $Z$ Pauli operators. The proof proceeds in three stages. In the first, we determine the effect of $M$, considered as a Clifford unitary operation, on a stabilizer state $|\psi\rangle$. This enables us, in the second stage, to find the action of the measurement projector $P_{a}=\left[I+(-1)^{a} M\right] / 2$ on $|\psi\rangle$ and thus to determine whether the measurement is certain or random. The post-measurement state is then found via a simple circuit identity in the last stage. Notice that, in determining the effect of $M$ on $|\psi\rangle$, we must retain the overall phase, since, in the second stage, the overall phase becomes a relative phase in the superposition $[|\psi\rangle+$ $\left.(-1)^{a} M|\psi\rangle\right] / 2$.

\section{Action of $Z$ on a stabilizer state}

To begin, consider the effect of a unitary $M$ on the $n$-qubit stabilizer state $|\psi\rangle$, where $M$ is known to be a tensor product consisting of only the operators $I$ and $Z$. The action of $I$ is trivial, so we can focus on determining the action of $Z$. As illustrated in Fig. 3(a), applying $Z$ to a solid node is equivalent to applying an $X$ operator to the same node prior to all other Clifford gates in the circuit. Similarly, the action of $Z$ on a hollow node can be reexpressed using the circuit identity in Fig. 3(c). This identity shows that an identical state is obtained by adding a leading $X$ operator to each neighbor of the hollow node and, if the hollow node has a sign, introducing an overall phase of -1 . For properly simplified measurements, hollow measured nodes are neighbored only by solid measured nodes, so only members of $\mathcal{M}_{\mathrm{S}}$, the measured solid nodes, collect leading $X$ operators. The number of $X$ operators collected by each member of $\mathcal{M}_{\mathrm{S}}$ is 1 plus the number of neighbors it has in the set $\mathcal{M}_{\mathrm{H}}$. Since $X^{2}=I$, the net result is that $X$ operators are added only to members of $\mathcal{M}_{\mathrm{SE}}$, the set of solid measured nodes with an even number of hollow measured neighbors.

Summarizing, the stabilizer state $M|\psi\rangle$ can be obtained from the state $|0\rangle^{\otimes n}$ by first applying an $X$ to each member of $\mathcal{M}_{\mathrm{SE}}$ and then applying the Clifford gates needed to obtain $|\psi\rangle$ from the initial state $|0\rangle^{\otimes n}$. In addition, an overall phase of $(-1)^{b}$ must be applied, where $b=\left|\mathcal{M}_{\mathrm{H}} \cap \mathcal{Z}\right|$ is the number of measured hollow nodes with a sign. That is,

$$
M|\psi\rangle=M U|0\rangle^{\otimes n}=(-1)^{b} U\left(\prod_{j \in \mathcal{M}_{\mathrm{SE}}} X_{j}\right)|0\rangle^{\otimes n}
$$

where $U$ is shorthand for the sequence of Clifford gates for preparing $|\psi\rangle=U|0\rangle^{\otimes n}$, as indicated by the stabilizer graph (see Eq. (2)).

\section{Certain and random outcomes}

The second stage of our proof applies this result to find the action of $P_{a}$ on $|\psi\rangle$ and the probability of obtaining measurement outcome $(-1)^{a}$, which is given by $\left\langle\psi\left|P_{a}\right| \psi\right\rangle$. We have immediately that

$$
\begin{aligned}
P_{a}|\psi\rangle & =\frac{1}{2}\left[I+(-1)^{a} M\right]|\psi\rangle \\
& =U \frac{1}{2}\left(I+(-1)^{a+b} \prod_{j \in \mathcal{M}_{\mathrm{SE}}} X_{j}\right)|0\rangle^{\otimes n},
\end{aligned}
$$

which gives

$$
\left\langle\psi\left|P_{a}\right| \psi\right\rangle=\frac{1}{2}\left(1+(-1)^{a+b}\left\langle\left. 0\right|^{\otimes n}\left(\prod_{j \in \mathcal{M}_{\mathrm{SE}}} X_{j}\right) \mid 0\right\rangle^{\otimes n}\right) .
$$


(a)
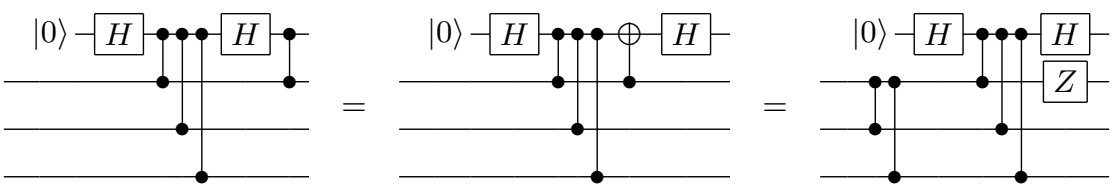

(b)
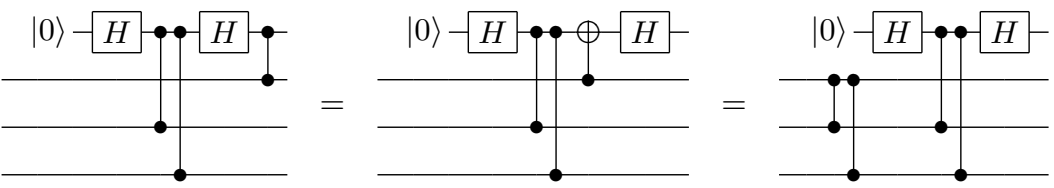

(c)
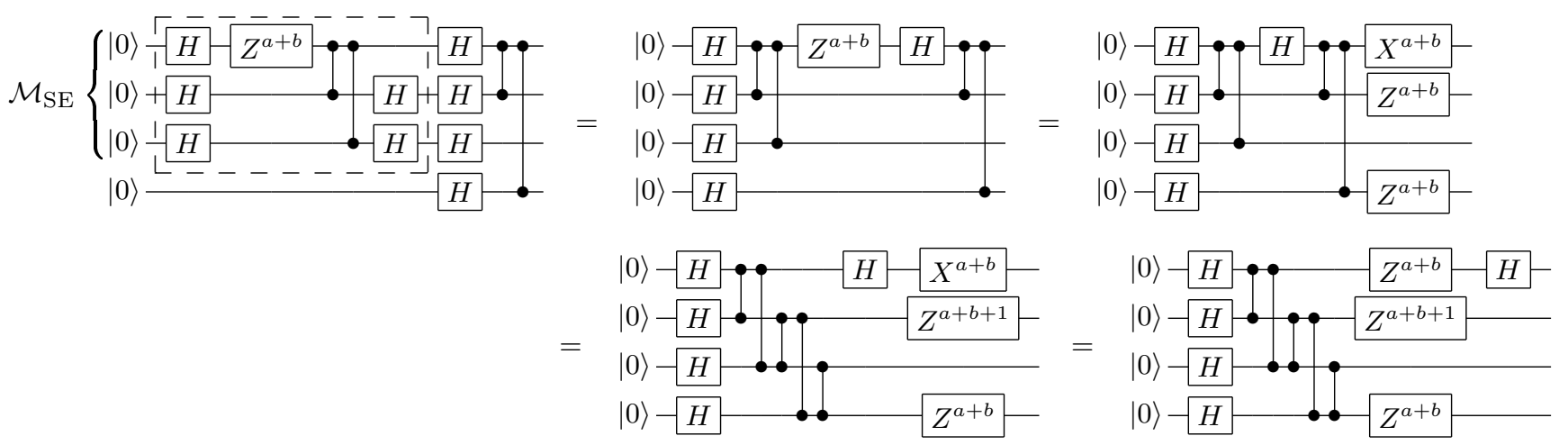

FIG. 4: Circuit identities used to determine the post-measurement state. Identities (a) and (b) utilize basic circuit identities for pushing a controlled-not gate past a controlled-sign gate. For the identity in (c), the first three qubits represent the elements of $\mathcal{M}_{\mathrm{SE}}$, with the chosen qubit $p$ placed on top. The dashed box delineates the Clifford operations that put the qubits in $\mathcal{M}_{\mathrm{SE}}$ into an appropriate cat state, i.e., those operations in Eq. (10) that are applied before $U$, whereas the gates after the dashed box are the relevant portion of the gates in $U$, i.e., the gates that create the original stabilizer state. The first equality in (c) is trivial. The second uses the identity in Fig. 3(b) to push $Z^{a+b}$ to the far right of the circuit, turning it into $X^{a+b}$ and depositing $Z^{a+b}$ on each neighbor of $p$. The next equality eliminates the controlled-sign gates that initially connected $p$ to other nodes by using the identity in (a) for connections to nodes in $\mathcal{M}_{\mathrm{SE}}$ and using (b) for connections to nodes outside of $\mathcal{M}_{\mathrm{SE}}$. The final simple equality returns the circuit to graph form.

Since $\langle 0|X| 0\rangle=0$, this means that measurements are classified into two types: if $\mathcal{M}_{\mathrm{SE}}=\varnothing$, the outcome probabilities are 1 , for $a=b(\bmod 2)$, and 0 , for $a \neq$ $b(\bmod 2)$, but if $\mathcal{M}_{\mathrm{SE}} \neq \varnothing, a$ has a $50 \%$ chance of being either 0 or 1 .

\section{Post-measurement state}

When the measurement outcome is $(-1)^{a}$, the postmeasurement state is $\left|\psi^{\prime}\right\rangle=P_{a}|\psi\rangle / \sqrt{\left\langle\psi\left|P_{a}\right| \psi\right\rangle}$. When $\mathcal{M}_{\mathrm{SE}}=\varnothing$, the outcome $a=b$ occurs with certainty, and the post-measurement state is the same as the original stabilizer state $|\psi\rangle$.

When $\mathcal{M}_{\mathrm{SE}} \neq \varnothing$, the situation is more complicated. In this case $\left\langle\psi\left|P_{a}\right| \psi\right\rangle=1 / 2$, so

$$
\left|\psi^{\prime}\right\rangle=U \frac{1}{\sqrt{2}}\left(I+(-1)^{a+b} \prod_{j \in \mathcal{M}_{\mathrm{SE}}} X_{j}\right)|0\rangle^{\otimes n} .
$$

Thus the post-measurement state is obtained by use of the Clifford circuit that created the original stabilizer state, but applied to an initial state that, for the qubits in $\mathcal{M}_{\mathrm{SE}}$, is changed to a cat state, i.e., an equal superposition of all 0 s and all 1s, with the sign between the two terms in the superposition given by $(-1)^{a+b}$. To construct a graph for the post-measurement state, we introduce a standard quantum circuit for making the cat state from $|0\rangle^{\otimes n}$ and use circuit identities to put the overall quantum circuit into graph form. Thus we write

$$
\begin{array}{r}
\left|\psi^{\prime}\right\rangle=U\left(\prod_{l \in \mathcal{M}_{\mathrm{SE} \backslash p}} H_{l}\right)\left(\prod_{k \in \mathcal{M}_{\mathrm{SE} \backslash p}}{ } Z_{p k}\right) \\
\times Z_{p}^{a+b} \prod_{j \in \mathcal{M}_{\mathrm{SE}}} H_{j}|0\rangle^{\otimes n},
\end{array}
$$

where $p$ denotes the chosen node. Figure 4 translates these Clifford operations into circuit notation and develops the identities needed to determine the postmeasurement state $\left|\psi^{\prime}\right\rangle$.

Most of the components of steps 1-4 in Sec. III B follow directly from Fig. 4(c), which begins with a circuit for a representative post-measurement state that is not in graph form. The boxed portion of the circuit puts the 
qubits in $\mathcal{M}_{\mathrm{SE}}$ into a cat state; that which follows is the relevant portion of the graph-form circuit for the premeasurement state. Application of a sequence of identities yields the circuit on the far right, which is in the proper form to translate to a stabilizer-state graph. From the first equality in Fig. 4(c), it can be seen that the new connections specified by rule 3 between $p$ and the unchosen nodes in $\mathcal{M}_{\mathrm{SE}}$ arise directly from the prepended cat state. The sign changes of $p$ 's neighbors given at the end of rule 2 follow from second equality. Pushing the Hadamard right of the remaining ${ }^{C} Z$ gates in the third equality and pushing the resultant ${ }^{C} X$ gates the other way removes $p$ 's previous connections while complementing edges between nodes that were neighbors of $p$ and the unchosen nodes in $\mathcal{M}_{\mathrm{SE}}$; these operations appear in rules 3 and 1, respectively. In the same equality, nodes that are both neighbors of $p$ and elements of the set $\mathcal{M}_{\mathrm{SE}}$ pick up the additional sign specified in the initial part of rule 2 . In the final equality, the chosen node becomes hollow, as specified in rule 3 and adopts a final sign of $(-1)^{a+b}$ as specified in rule 2 .

The final components of the measurement transformation rules deal with the cases in which the chosen node initially has a sign and/or a loop. The circuit identity in Fig. 4(c) does not explicitly cover these situations, but they are easily derived by applying the gates in question to either end of the identity and then applying the appropriate transformation rules to the right side. Rule 4 follows from transformation rule T3, while the alternative sign change in the initial part of rule 2 arises from transformation rule $\mathrm{T} 6$.
[1] M. B. Elliott, B. Eastin, and C. M. Caves, Phys. Rev. A 77, 042307 (2008).

[2] M. Hein, J. Eisert, and H. J. Briegel, Phys. Rev. A 69, 062311 (2004).

[3] D. Schlingemann, Quant. Inf. Comp. 4(4), 287 (2004).

[4] D. Schlingemann, Quant. Inf. Comp. 2(4), 307 (2002).

[5] M. Van den Nest, J. Dehaene, and B. De Moor, Phys.
Rev. A 69, 022316 (2004).

[6] S. Aaronson and D. Gottesman, Phys. Rev. A 70, 052328 (2004).

[7] R. Raussendorf and H. J. Briegel, Phys. Rev. Lett. 86, 5188 (2001). 